identify bio-medical and epidemiological factors that act as conduits for the transmission of HIV/AIDS and other STIs within crisis and unstable settings.

Methodology and Results It uses a multi-disciplinary approach that simultaneously embraces gender, poverty and biomedical analytical lenses to identify and strategically address emerging links between violence and HIV/AIDS. Interviews and VCT were administered to both multistage cluster and purposively-random sampled sexual violence victims in Kenya-A multiple logistic regression model controlling age, condom use (if any), and biological influences like the routes of exposure-whether vagino-penile or anal-penile-and sex of both victims and assailants was adopted. Overall prevalence for HIV, syphilis and gonorrhoea shot up: $2.3 \%, 1.1 \%$, and $3.1 \%$ respectively among men. For women: $2.7 \%, 1.9 \%$, and $4.2 .0 \%$ respectively.

Conclusion and Recommendations Sexual violence diminishes people's social capital in terms of social networks, norms, and traditions that could prove to be conducive to the spread of the HIV. We recommend early diagnosis to allow treatment of sexual violence survivors. Post Exposure Prophylaxis (PEP), to address exposure to HIV as a result of sexual violence, emergency contraception and VCT should form the backbone for intervention in order to address the menace of HIV/ AIDS. Anti-retroviral regimens dramatically improve rates of mortality and morbidity, prolong lives, improve quality of life, revitalise communities and transform perceptions of HIV/AIDS from a plague to a manageable, chronic illness.

\section{P2-S3.08 SELLING SEX ON THE DOORSTEP: DEVELOPMENT WORK FOR THE THIRD BRITISH NATIONAL SURVEY OF SEXUAL ATTITUDES AND LIFESTYLES (NATSAL 3)}

doi:10.1136/sextrans-2011-050108.327

${ }^{1} \mathrm{C}$ Tanton, ${ }^{2} \mathrm{~A}$ Phelps, ${ }^{2} \mathrm{~S}$ Nicholson, ${ }^{1} \mathrm{C}$ Mercer, ${ }^{1} \mathrm{P}$ Sonnenberg, ${ }^{3 \mathrm{~J}}$ Datta, ${ }^{3} \mathrm{~W}$ Macdowall, ${ }^{1} \mathrm{~B}$ Erens, ${ }^{3} \mathrm{~K}$ Wellings, ${ }^{1} \mathrm{~A}$ Johnson. ${ }^{1} \mathrm{UCL}$, London, UK; ${ }^{2}$ National Centre for Social Research, UK; ${ }^{3}$ London School of Hygiene \& Tropical Medicine, UK

Background The Third British National Survey of Sexual Attitudes \& Lifestyles (Natsal 3) builds upon groundbreaking research undertaken for Natsal 1 and Natsal 2 in 1990 and 2000, respectively. Natsal 3 aims to interview 15000 men and women aged 16-74 years during 2010-2012. However, the first pilot (Pilot 1) for Natsal 3 achieved a response rate of only $38.4 \%$, leading us to explore ways to maximise survey participation.

Methods Following Pilot 1, we consulted widely with interviewers, survey methodologists and communications experts to improve documents sent to households in advance of the interviewer calling, and the survey branding. This included simplifying the advance letter language and removing specific references to "sex"; developing a more detailed information leaflet to send with the letter; developing a study logo and respondent website. In Pilot 2, households were randomised to be sent the leaflet with the advance letter or to be given it when the interviewer called. We also examined the effect of the token of appreciation on participation rates by randomising households to receive either a $£ 15$ or a $£ 30$ voucher for participation. Results The overall response rate increased to 50.3\% (218/434 eligible addresses) in Pilot 2. This did not vary by whether or not households received the information leaflet in advance $(50.8 \%$ and $49.6 \%$, respectively), but interviewers felt that households sent the leaflet were more informed about the study and they were more confident approaching these households. Interviewers preferred the improved advance letter and some respondents reported that the website was a key factor encouraging them to participate. Pilot 2 response rates differed by token of appreciation (47.1\% vs $53.4 \%$ for $£ 15$ vs $£ 30$ ) but the sample size was too small to conclude that the difference was significant, so randomisation continued during the first wave of fieldwork. Response rates in wave 1 before reissuing were $48.4 \%$ vs $50.6 \%$ for $£ 15$ and $£ 30$, respectively ( $p=0.2$ ).

Conclusions Despite survey response rates declining generally, Natsal 3 development work has shown that it is possible to attain an adequate response in a population-based survey of sexual behaviour. Careful wording of participant documents, attractive survey branding and a participant website all contributed to increasing response rates. Response rates were slightly higher for the larger token of appreciation but increasing the value was not considered cost-effective.

\section{P2-S3.09 MOBILITY AS PREDICTOR OF INVOLVEMNET IN HIGH RISK SEXUAL RISK BEHAVIOUR}

doi:10.1136/sextrans-2011-050108.328

Z Kwena, C Obuya. Kenya Medical Research Institute, Kisumu, Kenya

Background Population mobility has long been associated with frequency and variety of sexually transmitted infections including HIV. People's mobility tends to disrupt their traditional social constraints and control of sexual behaviour by giving them opportunity, isolation and the desire for unique experiences. Fishermen being one of the highly mobile populations, we sought to evaluated the extent to their mobility predicts their involvement in high risk sexual behaviour.

Methods During a Phase I randomised double-blind placebocontrolled cross over trial evaluating the safety and acceptability of ethanol in emollient gel as a topical male microbicide, we collected data on fishermen's socio-economic and demographic characteristics, mobility patterns and sexual practices including extra-marital partnerships. We pre- and post-test counselled for STIs, obtained blood samples for HIV, HSV-2 and syphilis serologies and provided appropriate treatment and referrals. We analysed the data using descriptive statistics and then bivariate and multivariate logistic regression.

Results Of the 167 fishermen screened, over a half (52\%) were mobile defined as travelling and spending at least one night away from home in the month preceding the study). Two-thirds (63\%) had active extra-marital relationships with only $5 \%$ consistently using condoms in these sexual encounters. During travel, a quarter (24\%) drank alcohol and a similar number $(25 \%)$ had sex with a third (32\%) reporting new casual sex partners. At bivariate level, those travelling in the month preceding the study were more likely to be HIV+ (OR 2.08; 95\% CI 1.01 to 4.28), have an active extramarital relationship (OR 3.69; 95\% CI 1.57 to 8.68), and have multiple sex partners in the six month preceding the study (OR 2.04; 95\% CI 1.06 to 3.95). Mobility among fishermen was independently associated with having an active extra-marital relationship (AOR 3.64; 95\% CI 1.11 to 12.00).

Conclusion Mobile fishermen exhibit high risk sexual behaviour that include extra-marital sex and low condom use. This population is likely to benefit from STI/HIV prevention intervention.

\section{P2-S3.10 HSV-2 SEROLOGIC TESTING AND PSYCHOSOCIAL HARM: A SYSTEMATIC REVIEW}

doi:10.1136/sextrans-2011-050108.329

K Ross, A Wald, C Johnston. University of Washington, Seattle, USA

Background Serologic testing for herpes simplex virus type-2 (HSV2 ) in persons without a history of genital herpes is currently not recommended partly due to the concern that HSV-2 diagnosis would lead to negative psychosocial sequelae, such as anxiety and depression. We conducted a systematic review to assess the evidence 